\title{
Jellyfish envenomation: A chilling toxidrome of seizures and cyanosis - a case report
}

\author{
S Mundkur, ${ }^{1}$ MBBS, DCH, DNB; S Shashidhara, ${ }^{1}$ MBBS, MD; S Hebbar, ${ }^{1}$ MBBS, MD; A Kumar, ${ }^{2}$ MBBS, MD, \\ S Kanaparthi, ${ }^{1}$ MBBS, MD \\ ${ }^{1}$ Department of Pediatrics, Women and Child Block, Kasturba Medical College, Madhav Nagar, Manipal Academy of Higher Education, \\ Manipal, India \\ ${ }^{2}$ Department of Forensic Medicine, Kasturba Medical College, Madhav Nagar, Manipal University, Manipal, India
}

Correspondingauthor: S Shashidhara (sowmya.s@manipal.edu)

\begin{abstract}
Jellyfish envenomation is a common incident in coastal areas all over the world. While the majority of reported cases are self-limiting with few lasting complications, a few deadly species can cause life-threatening and debilitating illnesses with a prolonged recovery time. Chrysaora spp. have been known to cause a diverse spectrum of manifestations. We report the case of a 7-year-old boy with jellyfish envenomation presenting with cyanosis, seizures and hypertension not previously described in association with the Chrysaora spp.
\end{abstract}

S Afr J Child Health 2018;12(2):73-75. DOI:10.7196/SAJCH.2018.v12i2.1429

Jellyfish are intriguing and are found ubiquitously in all oceans. However, species predominance is seen across different marine ecosystems. The most common species seen across coastal India are Aurelia and Physalia. ${ }^{[1]}$ Chrysaora spp. are widely dispersed across the Atlantic, Pacific and Indian oceans. ${ }^{[2]}$ Most Chrysaora spp. envenomations are mild, with irritation of the skin and pain, requiring outpatient medical attention..$^{[3]}$ These species have rarely been reported to cause neurotoxicity or digital ischaemia, although they are an expected complication of the more toxic species. ${ }^{[4-6]}$ In this case report, we describe a rare case of a Chrysaora spp. sting associated with dermatitis, neurotoxicity and vasospasm leading to hypertension.

\section{Case report}

A 7-year-old boy of European origin who was on vacation in coastal Karnataka, India, was brought to triage with complaints of a jellyfish sting belonging to species Chrysaora while bathing in the sea, resulting in a rash over both his hands and legs which had been progressively increasing over 12 hours. The parents described the rash as spread out over the sites of attachment of the tentacles of the jellyfish which had to be forcibly separated from the boy. Swelling of the right knee was subsequently noted by the parents, along with pain and a progressive bluish discolouration of the third, fourth and fifth fingers on his right hand.

His past medical history was unremarkable and he was developmentally healthy and appropriately immunised for his age. His other family members were healthy and unaffected. He was seen by a primary care physician $10-12$ hours after the sting and was referred to our institution.

The boy was observed at our triage after 15 hours after the sting. $\mathrm{He}$ was conscious and oriented at the time and the following vitals were recorded: Glasgow Coma Scale score, 15/15; heart rate, 92 beats per minute; blood pressure, 104/60 (95th centile - 117/78); and respiratory rate, 16 breaths per minute. On examining the peripheral pulses, the right radial pulse was feeble with small volume; all other pulses were well felt. Spo2-right upper limb was not recordable. Left upper limb: 98\%; right lower limb: 96\%; left lower limb: $98 \%$. The third, fourth and fifth digits of the right hand were cyanosed and swollen, with painful movements up to the proximal interphalangeal joints including the nail beds (Fig. 1). An erythematous maculopapular rash was present on both thighs, legs and forearms, and flagellate pigmentation was present over both thighs. Purpuric lesions over the right thigh and popliteal fossa, with a local rise in temperature and extreme pain on movement were also seen (Fig. 2). The respiratory, abdominal and cardiovascular examinations were normal. Neurological examination at admission showed no focal neurological deficits.

The patient's laboratory parameters were as follows: alanine transaminase $13.0 \mathrm{IU} / \mathrm{L}$; aspartate transaminase, $45.0 \mathrm{IU} / \mathrm{L}$; C-reactive protein, $1.4 \mathrm{mg} / \mathrm{L}$; serum creatinine, $0.4 \mathrm{mg} / \mathrm{dL}$; serum potassium, $4.6 \mathrm{mmol} / \mathrm{L}$; serum sodium, $134.0 \mathrm{mmol} / \mathrm{L}$; serum urea, $25 \mathrm{mg} / \mathrm{dL}$; erythrocyte sedimentation rate, $9 \mathrm{~mm} / \mathrm{hr}$; haemoglobin, $13.6 \mathrm{~g} / \mathrm{dL}$; haematocrit, $41.8 \%$; mean corpuscular volume, $82.8 \mathrm{fl}$; platelet count, $299.0 \times 10^{3} / \mu \mathrm{L}$; red blood cell count, $5.06 \times 106 / \mu \mathrm{L}$; RDW, $14.0 \%$; total white blood cells, $15.8 \times 10^{3} / \mu \mathrm{L}$; serum calcium, $9.3 \mathrm{mg} / \mathrm{dL}$; serum chloride, $93.8 \mathrm{mmol} / \mathrm{L}$; serum magnesium, $2.0 \mathrm{mg} / \mathrm{dL}$; serum phosphorus, $5.7 \mathrm{mg} / \mathrm{dL}$; complement C3 level, $131.0 \mathrm{mg} / \mathrm{dL}$; complement C4 level, $19.0 \mathrm{mg} / \mathrm{dL}$; anti-nuclear antibody profile, negative; urine examination: normal.

The right upper limb arterial Doppler showed attenuation of the axillary, brachial, radial and ulnar arteries with a regular colour flow, flow velocities and spectral waveforms with low biphasic resistance and diffuse subcutaneous oedema. The renal artery Doppler and 2D

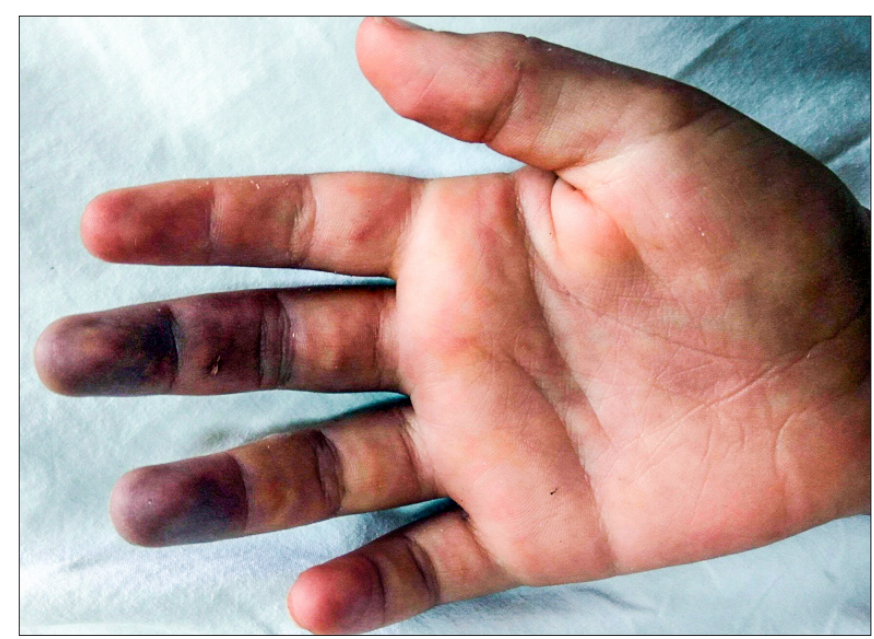

Fig. 1. Digital ischaemia of the fingertips following jellyfish sting. 


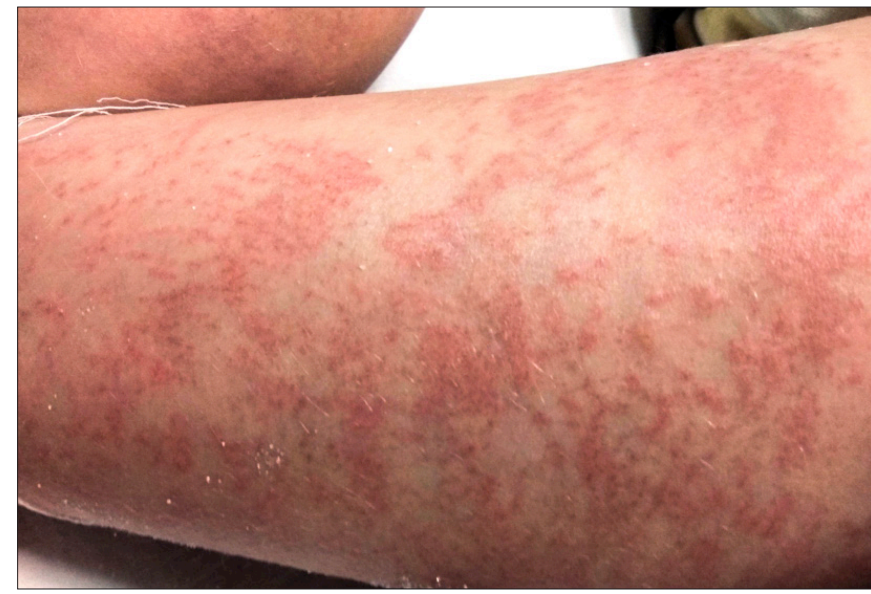

Fig. 2. Papular flagellate rash at the site of attachment of the jellyfish tentacles.

echocardiograph were normal. An ultrasound demonstrated diffuse subcutaneous oedema with moderate knee-joint effusion with septation. Computed tomography angiography showed attenuation in the calibre of the distal two-thirds of the right brachial artery, radial and ulnar arteries and faint contrast opacification (Fig. 3).

Treatment was initiated with subcutaneous enoxaparin, intravenous dexamethasone, oral ecospirin and oral cilostazol. On the third day following the sting, the patient developed five episodes of generalised tonic-clonic seizures which required levetiracetam $(40 \mathrm{mg} / \mathrm{kg} /$ day $)$ and valproate $(10 \mathrm{mg} / \mathrm{kg} /$ day). Magnetic resonance imaging of the brain revealed patchy bilateral asymmetrical T2/ FLAIR hyperintensities involving bilateral frontal-parietooccipital sulci in the cortex predominantly, with mild extension into the adjacent subcortical white matter and focal extension into the deep white matter (Fig. 4). Electroencephalography indicated a generalised disturbance of electrical function. The boy was found to be hypertensive on the fourth day, with a blood pressure reading of $140 / 100$. The hypertension was controlled with oral nifedipine. The rash over the arms worsened over the course of five days and required therapy with topical antibiotics, antihistamines and steroids. The right knee effusion was treated conservatively.

The reappearance of the right radial pulse and reperfusion of the digits was observed after 7 days. He remained normotensive and seizure-free after the 8th day. However, a right-sided foot drop was noted on the 10th day, which responded minimally to therapy and rehabilitation. A residual reddish hue persisted as a remnant of the rash. He was normotensive and seizure-free on maintenance antiepileptics at discharge with a right foot drop.

\section{Discussion}

Jellyfish belong to the phylum Cnidaria, which has 10000 species and only $\sim 100$ of those are toxic to humans. ${ }^{[7]}$ Although only a few species contain tentacles, nematocysts are found in all. The length of the nematocysts in the vast majority are insufficient to penetrate the dermis and are therefore harmless. ${ }^{[8]}$ Upon skin contact, the toxin produced by a number of species causes a local reaction involving the phospholipase A2 pathway, which leads to pain, swelling and skin necrosis in severe conditions. ${ }^{[9]}$ Once in the general circulation, the toxin has gastrointestinal, cardiac, neurological, muscular and immunogenic effects. The venom also contains haemolytic and lethal fractions affecting the heart. ${ }^{[2]}$

Jellyfish stings most frequently result in pain, itching, an intense burning sensation and redness in the majority of cases. However, a few studies show that allergic reactions and anaphylaxis are remote complications as the jellyfish toxin is known to act by inducing the release of inflammatory mediators. ${ }^{[10]}$ The classical jellyfish dermatitis, also called seabather's eruption, is believed to occur

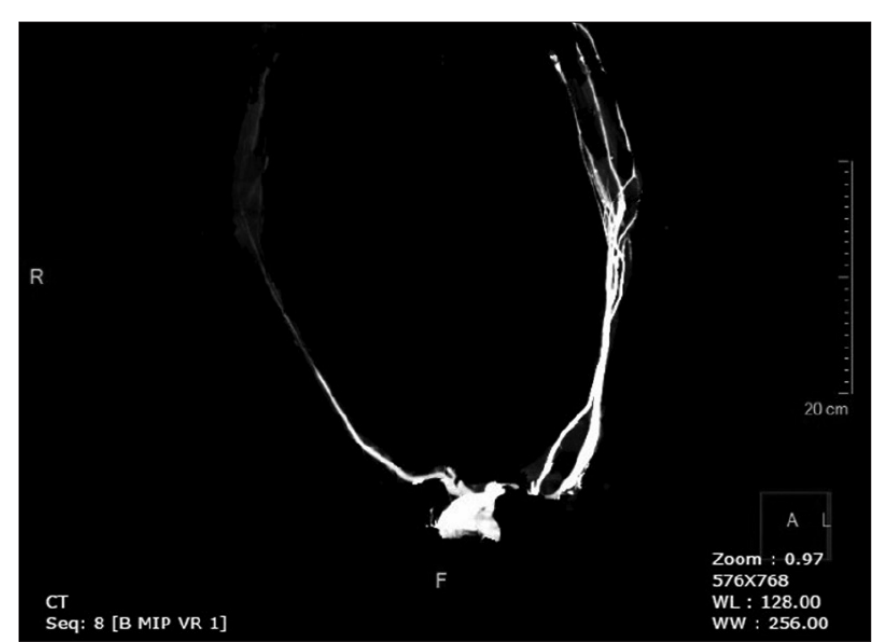

Fig. 3. Computed tomography angiograph showing narrowing and spasm of the right brachial, radial and ulnar arteries.

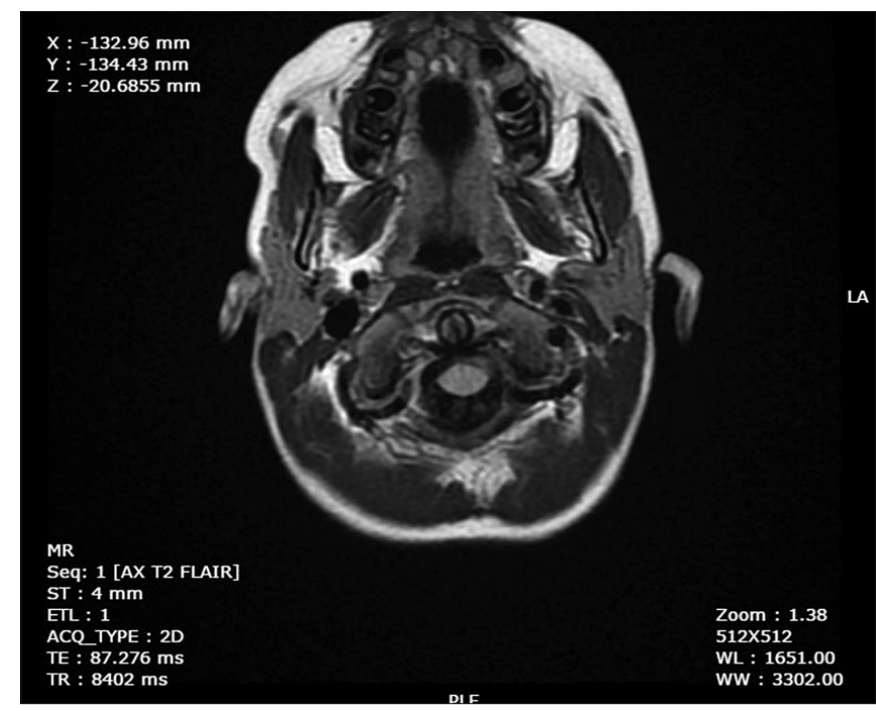

Fig. 4. Magnetic resonance imaging of the brain: T2 weighted image showing hyperintensities of the fronto-parieto-occipital cortices.

due to the extended action of the jellyfish toxin leading to intense burning, pain, itching and erythematous papules for a prolonged time after the exposure. ${ }^{[11,12]}$ Synonymous with this, the patient in our case presented as described and responded to topical corticosteroids and antihistamines.

The venom of the Chrysaora spp. has been demonstrated to cause calcium ion influx through voltage-gated channels and cause severe arterial spasm in experimental studies. ${ }^{[4]}$ Jellyfish envenomation leads to acute regional vasospasm leading to cyanosis of the affected areas and absence of sweating and piloerection ${ }^{[6,13]}$ Chrysaora spp. causing these effects have been reported in rare cases across the Indian coast. ${ }^{[5,6}$ Analogously, our patient developed a delayed response to the sting with a severe spasm of the right brachial artery, swelling, pain and cyanosis of the right third and fourth digits. Sympathomimetic action of the venom is known to cause severe hypertension in a syndrome commonly called the Irukandji, which is accompanied by severe cardiotoxic manifestations mimicking a catecholamine crisis. ${ }^{[14]}$ Hypertension, as described in these studies, was noted in our patient who was controlled with a direct arteriolar dilator such as cilostazol, a phosphodiesterase inhibitor, and nifedipine, a calcium channel blocker.

The toxin isolated from nematocysts of Chrysaora is known to depolarise nerve and muscle membranes and to increase the frequency 
of miniature end-plate potentials. ${ }^{[15]}$ Motor and sensory neurotoxic effects of cnidarian venoms have been reported in several cases of mononeuritis multiplex produced by different jellyfish. ${ }^{[16]}$ There have also been reports of Guillain-Barré syndrome and dysphonia following jellyfish stings. ${ }^{[17-19]}$ However, Chrysaora stings leading to neurological complications are seldom seen. Our patient developed seizures three days following exposure. Patchy bilateral hyper intensities were seen over the cortical areas on MRI. A point to consider here is that these changes may be due to a postictal state. However, after ten days he had a residual foot drop on the right side.

Treatment of jellyfish stings should begin by administration of basic life support. Appropriate methods for tentacle removal, such as flushing with sea water, using tweezers or scraping off with a card, should be done carefully as they carry the risk of further discharge of nematocysts. ${ }^{[20]}$ The application of vinegar and an ice pack to the local area to reduce pain is recommended. The affected part should then be immersed in water as hot as is bearable if no proprietary meds are available. Skin inhibitor creams are commercially available which provide protection for swimmers against jellyfish stings. ${ }^{[21]}$

\section{Conclusion}

Jellyfish envenomation is usually harmless or causes mild illness. However, in rare instances, it can manifest as a toxidrome of seizures, cyanosis and hypertension due to prolonged and delayed multisystem effects of the toxin, requiring continuous intensive monitoring during the acute phase. Residual neurological deficits can be anticipated following envenomation. Adequate awareness by seabathers, personal protection, and prompt medical intervention is necessary to prevent morbidity.

\footnotetext{
Acknowledgements. Grieselda Philomena Noronha and Varna Shetty are gratefully acknowledged for the $\mathrm{CT}$ angiography and MRI reports.

Author contributions. SS, SM, SH, SK: primary management of the patient, co-authored the manuscript and contributed to final editing. AK: assisted in species identification and toxicological aspects of the case, as well as drafting and editing of the manuscript.

Funding. None.

Conflicts of interest. None.
}

1. Venkataraman KR, Raghunathan C, Sreeraj CR Raghuraman R. Guide to the Dangerous and Venomous Marine Animals of India. Kolkata: Zoological Survey of India, 2012:1-98
2. Cegolon L, Heymann WC, Lange JH, Mastrangelo G. Jellyfish stings and their management: A review. Mar Drugs 2013;11(2):523-550. https://doi. org/10.3390/md11020523

3. Williamson JA, Fenner PJ, Burnett JW. The injuries, their incidence and the toxins that produce them. In: JA Williamson, Fenner PJ, Burnett JW, Rifkin JF, eds. Venomous and Poisonous Marine Animals: A Medical and Biological Handbook. Sydney: New South Wales University Press, 1996:63-87.

4. Burnett JW, Weinrich D, Williamson JA, Fenner PJ, Lutz LL, Bloom DA Autonomic neurotoxicity of jellyfish and marine animal venoms. Clin Auton Res 1998;8(2):125-130.

5. Hach-Wunderle V, Mebs D, Frederking K, Breddin HK. Jellyfish poisoning. Deutsch Med Wochenschr 1987;112(48):1865-1868. https://doi. org/10.1055/s-2008-1068344

6. Williamson JA, Burnett JW, Fenner PJ, Hach-Wunderle V, Hoe LY, Adiga KM. Acute regional vascular insufficiency after jellyfish envenomation. Med J Aust 1988;149(11-12):698-701.

7. Kramp PL. Synopsis of the medusae of the world. J Marine Biol Assoc UK 1961;40:7-382. https://doi.org/10.1017/S0025315400007347

8. Barnes JH. Observations on jellyfish stingings in North Queensland. Med J Aust 1960;47(2):993.

9. Mariottini GL, Pane L. Mediterranean jellyfish venoms: A review on Scyphomedusae. Marine Drugs 2010;8(4):1122-1152. https://doi.org/10.3390/ md8041122

10. De Donno A, Idolo A, Bagordo F, Grassi T, Leomanni A, Serio F. Impact of stinging jellyfish proliferations along south Italian coasts: Human health hazards, treatment and social costs. Int $\mathrm{J}$ Environ Res Public Health 2014;11(3):2488-2503. https://doi.org/10.3390/ijerph110302488

11. Rossetto AL, Da Silveira FL, Morandini AC, Haddad V, Resgalla C. Seabather's eruption: Report of fourteen cases. An Acad Bras Cienc 2015;87(1):431-436. https://doi.org/10.1590/0001-3765201520130468

12. Raupp U, Milde P, Goerz G, Plewig G, Burnett J, Heeger T. Case report of jellyfish injury. Hautarzt 1996;47(1):47-52.

13. Binnetoglu FK, Kizildag B, Topaloglu N, Kasapcopur O. Severe digital necrosis in a 4-year-old boy: Primary Raynaud's or jellyfish sting. BMJ Case Rep 2013;2013. https://doi.org/10.1136/bcr-2013-201478

14. Bianchi R, Torella D, Spaccarotella C, Mongiardo A, Indolfi C. Mediterranean jellyfish sting-induced Tako-Tsubo cardiomyopathy. Eur Heart J 2011;32(1):18. https://doi.org/10.1093/eurheartj/ehq349

15. Dubois JM, Tanguy J, Burnett JW. Ionic channels induced by sea nettle toxin in the nodal membrane. Biophys J 1983;42(2):199-202.

16. Burnett JW WJ, Fenner PJ. Mononeuritis multiplex after coelenterate sting. Med J Australia 1994;161:321-322.

17. Devere R. Guillain-Barre syndrome after a jellyfish sting. J Clin Neuromuscul Dis 2011;12(4):227. https://doi.org10.1097/CND.0b013e3181elf046

18. Pang KA, Schwartz MS. Guillain-Barre syndrome following jellyfish stings (Pelagia noctiluca). J Neurol Neurosurg Psychiatry 1993;56(10):1133.

19. Burnett JW. Dysphonia: A new addition to jellyfish envenomation syndromes. Wilderness Environ Med 2005;16(2):117-118.

20. Ward NT, Darracq MA, Tomaszewski C, Clark RF. Evidence-based treatment of jellyfish stings in North America and Hawaii. Ann Emerg Med 2012;60(4):399414. https://doi.org/10.1016/j.annemergmed.2012.04.010

21. Tonseth KA, Andersen TS, Pripp AH, Karlsen HE. Prophylactic treatment of jellyfish stings--a randomised trial. Tidsskr Nor Laegeforen 2012;132(1213):1446-1449. https://doi.org/10.4045/tidsskr.11.0652 